\title{
Estimativas de Herdabilidade para Perímetro Escrotal de Animais da Raça Nelore ${ }^{1}$ Laila Talarico Dias², Lenira El Faro ${ }^{3}$, Lúcia Galvão de Albuquerque ${ }^{4}$
}

\begin{abstract}
RESUMO - Estimaram-se parâmetros genéticos e de ambiente para perímetro escrotal ao sobreano (PE) de 9.355 animais da raça Nelore. Foram considerados no modelo o efeito aleatório de animal e os efeitos fixos de grupo contemporâneo (GC) e os efeitos linear e quadrático das covariáveis idade ao sobreano (IDS) e peso ao sobreano ajustado aos 550 dias de idade (PSAJ). O grupo contemporâneo foi definido pelas variáveis: ano, estação e fazenda de nascimento, grupo de manejo ao nascimento, desmama e sobreano. As estimativas de herdabilidade foram 0,42 $\pm 0,04$, quando considerados PSAJ e IDS como covariáveis; 0,41 \pm 0,04, considerando-se PSAJ como covariável; e $0,35 \pm 0,03$, com apenas IDS como covariável.
\end{abstract}

Palavras-chave: bovino de corte, herdabilidade, precocidade sexual

\section{Heritability Estimates for Scrotal Circumference in Nelore Cattle}

\begin{abstract}
Genetic parameters and environmental effects were estimated for 9,355 Nelore cattle scrotal circumference measurements (PE). The univariate analysis considered animal effect (random effect) and fixed effects of contemporary group (GC) and linear and quadratic effects of age at yearling (IDS) and yearling weight adjusted to 550 days (PSAJ). Contemporary group was defined by: year, season at birth and management group at birth, weaning and yearling. Heritability estimates were: $0.42 \pm 0.04$ (after IDS and PSAJ); $0.41 \pm 0.04$ (after PSAJ) and $0.35 \pm 0.03$ (adjusting only for IDS).
\end{abstract}

Key Words: beef cattle, heritability, sexual precocity

\section{Introdução}

O perímetro escrotal é uma característica que vem sendo utilizada nos programas de melhoramento genético animal, pois segundo diversos autores, esta é uma medida que pode ser obtida facilmente, é altamente herdável, além de estar correlacionada com características de crescimento e de reprodução (Latimer et al., 1982; Alencar et al., 1993; Brinks et al., 1994; Brito, 1997). Entretanto, Brito (1997) sugeriu que as medidas de perímetro escrotal deveriam ser padronizadas para uma mesma idade ou peso corporal, pois, dessa forma, seria possível obter progressos genéticos em precocidade sexual, sem acarretar, necessariamente, aumento no tamanho adulto dos animais.

Teixeira et al. (1998) sugerem que seria importante que os programas de melhoramento genético oferecessem aos produtores as medidas de perímetro escrotal corrigidas para peso e idade e também as medidas corrigidas apenas para idade, pois podem haver diferenças de prioridade em relação à seleção para precocidade sexual e precocidade de crescimento.
O objetivo do presente trabalho foi estimar os parâmetros genéticos e de ambiente para perímetro escrotal ao sobreano de animais da raça Nelore.

\section{Material e Métodos}

Foram utilizados dados de animais da raça Nelore, pertencentes à Agropecuária Jacarezinho Ltda., situada no município de Valparaíso - SP. O arquivo de dados continha informações de animais nascidos entre 1984 e 1997, totalizando 9.355 registros de perímetro escrotal (PE).

A definição de grupo contemporâneo incluiu as variáveis: ano e estação de nascimento, fazenda e grupo de manejo ao nascimento, à desmama e ao sobreano, totalizando 382 grupos contemporâneos distintos.

Foram estabelecidas duas estações de nascimento, sendo que foi determinado como estação 1 os animais nascidos entre os meses de janeiro e junho e estação 2 os animais nascidos entre os meses de julho e dezembro.

Como covariáveis foram considerados os efeitos

\footnotetext{
1 Parte da Dissertação de Mestrado em Genética e Melhoramento Animal da FCAV - UNESP/Jaboticabal, financiada pela CAPES.

2 Zootecnista - Doutoranda em Zootecnia da FCAV - UNESP/Jaboticabal. E.mail: laila@fcav.unesp.br

${ }^{3}$ Pesquisador Científico do Instituto de Zootecnia de Ribeirão Preto - SP (APTA).

${ }^{4}$ Professora Adjunto do Departamento de Zootecnia - FCAV - UNESP. Pesquisadora do CNPq. E.mail: Igalb@fcav.unesp.br
} 
de idade ao sobreano (IDS) e peso ao sobreano ajustado para 550 dias de idade (PSAJ).

Para a modelagem dos efeitos fixos, foram utilizados três modelos.

O modelo 1 é representado por:

$y_{i j}=\mu+G C_{i}+b_{1}\left(I D S_{i j}-\overline{I D S}\right)_{+}+b_{2}\left(I D S_{i j}-\overline{I D S}\right)^{2}+b_{3}\left(P S A J_{i j}-\overline{P S A J}\right)+b_{4}\left(P S A J_{i j}-\overline{P S A J}\right)^{2}+\varepsilon_{i j}$ em que: $\mathrm{y}_{\mathrm{ij}}=$ valor observado do perímetro escrotal ao sobreano do animal $\mathrm{j}$, pertencente ao iésimo grupo contemporâneo; $\mu=$ média geral das medidas de perímetro escrotal; $\mathrm{GC}_{\mathrm{i}}=$ efeito do iésimo grupo contemporâneo; $\operatorname{IDS}_{\mathrm{ij}}=$ idade ao sobreano do animal $\mathrm{j}$, pertencente ao iésimo grupo contemporâneo; $\overline{I D S}=$ média de idade ao sobreano; PSAJ $_{\mathrm{ij}}=$ peso ao sobreano ajustado aos 550 dias de idade do animal $\mathrm{j}$, pertencente ao iésimo grupo contemporâneo;

$\overline{P S A J}=$ média de peso ao sobreano ajustado aos 550 dias de idade; $b_{1}=$ coeficiente de regressão linear da idade ao sobreano; $b_{2}=$ coeficiente de regressão quadrático da idade ao sobreano; $b_{3}=$ coeficiente de regressão linear do peso ao sobreano ajustado aos 550 dias de idade; $b_{4}=$ coeficiente de regressão quadrático do peso ao sobreano ajustado aos 550 dias de idade; $\mathrm{e}_{\mathrm{ij}}=$ erro aleatório associado a cada observação.

O modelo 2, semelhante ao modelo1, excluindo os efeitos linear e quadrático de IDS.

O modelo 3, semelhante ao modelo 1, excluindo os efeitos linear e quadrático de PSAJ.

As análises foram realizadas pelo método dos quadrados mínimos, utilizando-se o procedimento GLM do SAS (SAS, 1998).

Após o ajuste do PE para o efeito de GC, obtidos do modelo completo, modelo 1, utilizou-se uma análise de regressão (Procedimento REG do SAS), apenas com as covariáveis, para estimar o fator de inflação de variância e verificar possíveis ocorrências de multicolinearidade. $\mathrm{O}$ fator de inflação de variância (FIV) é o aumento da variância dos $\beta^{\prime}$ 's, determinado pela correlação entre as variáveis independentes, cuja magnitude é da ordem de $\left[1 /\left(1-R^{2}\right)\right]$, em que $\mathrm{R}^{2}$ é o coeficiente de determinação (Freund \& Wilson, 1998). Esse procedimento foi realizado porque, quando ocorre multicolinearidade, os testes estatísticos podem falhar em detectar diferenças significativas entre os fatores.

Para a estimação dos componentes de variância, os dados foram analisados por máxima verossimilhança restrita (Patterson \& Thompson, 1971) empregando-se o método não derivativo descrito por
Smith \& Graser (1986) e Graser et al. (1987). Foram aplicados modelos animais utilizando o algorítmo desenvolvido por Boldman et al. (1991, 1993) disponível no pacote Multiple trait derivative-free maximum likelihood - MTDFREML.

Para essas análises foi incluído o efeito aleatório de animal, além dos efeitos fixos anteriormente citados.

O modelo geral utilizado pode ser representado na forma matricial como:

$$
\mathrm{y}=\mathrm{X} \beta+\mathrm{Za}+\mathrm{e}
$$

em que: $y=$ vetor de variáveis dependentes; $\beta=$ vetor de efeitos fixos; $\mathrm{a}=$ vetor de valores genéticos aditivos dos animais; $\mathrm{e}=$ vetor de efeitos residuais; $\mathrm{X}$ e $\mathrm{Z}$ são matrizes incidência respectivas para cada efeito.

Foram assumidas as seguintes pressuposições:

$$
\begin{gathered}
\mathrm{E}(\mathrm{y})=\mathrm{X} \beta, \mathrm{E}(\mathrm{a})=0 \text { e } \mathrm{E}(\mathrm{e})=0 ; \\
\operatorname{Var}\left[\begin{array}{l}
a \\
e
\end{array}\right]=\left[\begin{array}{ll}
G & 0 \\
0 & R
\end{array}\right]
\end{gathered}
$$

em que: $\mathrm{G}=$ matriz de variância genética aditiva, obtida por $\mathrm{A} \sigma_{\mathrm{a}}^{2} ; \mathrm{A}=$ matriz de parentesco; $\mathrm{R}=$ matriz de variância residual, obtida por $\mathrm{I} \sigma_{\mathrm{e}}{ }^{2}$; $\mathrm{I}=$ matrizidentidade; $\sigma_{\mathrm{a}}{ }^{2}=$ variância genética aditiva direta; $\sigma_{\mathrm{e}}^{2}=$ variância residual.

Os componentes de variância foram estimados utilizando-se os mesmos efeitos fixos dos três modelos anteriormente citados.

\section{Resultados e Discussão}

As médias obtidas para perímetro escrotal, idade ao sobreano e peso ao sobreano ajustado aos 550 dias de idade foram, respectivamente, $26,33 \mathrm{~cm} ; 545,09$ dias e $319,71 \mathrm{~kg}$. Valores semelhantes foram encontrados por Pereira et al. (1998), que, ao trabalharem com dados de animais da raça Nelore, observaram valores médios para perímetro escrotal ao sobreano de $26,7 \mathrm{~cm}$ e peso corporal ao sobreano de $314,5 \mathrm{~kg}$. Dal-Farra et al. (1998) também obtiveram resultados próximos aos do presente trabalho. Os autores relataram médias para perímetro escrotal ao sobreano, idade ao sobreano e peso ao sobreano ajustado para 550 dias de 28,44 cm, 589,04 dias e $333,03 \mathrm{~kg}$, respectivamente.

Os resultados das análises de variância e regressão do perímetro escrotal estão apresentados na Tabela 1.

Pode-se observar que o efeito de GC influenciou significativamente $(\mathrm{P}<0,01)$ o $\mathrm{PE}$ em todos os modelos utilizados. 
De acordo com o modelo 1, os efeitos linear e quadrático de idade ao sobreano (IDS), bem como o efeito quadrático do peso ao sobreano ajustado (PSAJ), não influenciaram significativamente o perímetro escrotal (PE). Estes resultados não concordam com os de Dal-Farra et al. (1998), os quais verificaram que tanto o efeito linear e quadrático da idade como o do peso ao sobreano influenciaram significativamente o perímetro escrotal dos animais da raça Nelore. Entretanto, Ortiz-Peña (1998) não encontrou influência do efeito quadrático da idade sobre o perímetro escrotal de animais da raça Nelore.

No presente estudo, quando apenas o peso foi considerado como covariável (modelo 2), constatouse que o efeito linear afetou significativamente o PE. Com relação ao modelo que continha apenas a idade como covariável (modelo 3), não foi observada influência significativa sobre o PE.

A não significância do efeito da idade sobre o PE pode ter ocorrido devido a diferentes fatores. Verificou-se que em mais de $65 \%$ dos GC's os animais tinham diferenças de idade de, no máximo, 30 dias (Figura 1). Esta pequena variação da idade dentro de GC era esperada devido às práticas de manejo da fazenda, formando grupos de manejo bastante homogêneos. Na Figura 1, é possível observar que em, aproximadamente, $20 \%$ dos GC's existentes a diferença de idade entre os animais dentro de um mesmo GC não ultrapassa 10 dias e que em torno de $45 \%$ dos GC's tal diferença não supera 20 dias.

Outro fator que pode ter contribuído para que os efeitos de IDS e PSAJ (quadráticos) sobre o PE não fossem significativos (modelo 1) foi a correlação entre esses efeitos. Utilizando-se o procedimento REG, após o ajuste dos dados de PE para as soluções de GC, verificaram-se valores de FIV variando de 162,84 a 478,96 (Tabela 1). De acordo com Freund e Wilson (1998), valores de FIV maiores do que 10 indicam sérios problemas de multicolinearidade. Isto sugere que, embora o efeito da idade seja biologicamente importante e explique parcela ponderável da variação de PE, devendo ser considerado ao realizar a avaliação genética dos animais, nem sempre é possível detectar efeito estatisticamente significativo da idade ao sobreano sobre o PE.

Ainda assim, os valores preditos de PE mostram diferença de até 4,6 cm entre os animais mais velhos e os mais jovens (Figura 2). Da mesma forma, a maior diferença dos valores do PE preditos, para o peso ao sobreano, entre os animais mais leves e os mais pesados ao sobreano foi de $6,2 \mathrm{~cm}$ (Figura 3). Esses resultados mostram que esses dois efeitos foram importantes fontes de variação para o $\mathrm{PE}$ e não podem ser ignorados.

Na Tabela 2, estão apresentadas as estimativas dos componentes de variância para o PE, para os três modelos considerados. Vale ressaltar que para essas análises foi incluído o efeito aleatório de animal, além dos efeitos fixos citados anteriormente.

Pode-se observar que, no modelo 1 , o qual considerou os efeitos de IDS e de PSAJ, a estimativa de herdabilidade obtida foi superior a obtida com o modelo 3 (com apenas IDS como covariável), pois houve aumento da variância genética aditiva de $2,56 \%$

Tabela 1 - Resumo das análises de variância e da análise de regressão do perímetro escrotal de acordo com diferentes modelos

Table 1 - Analyses of variance and regression for scrotal circumference, according to the three models

\begin{tabular}{lccccc}
\hline & $\begin{array}{c}\text { Modelo 1 } \\
\text { Model 1 }\end{array}$ & $\begin{array}{c}\text { Modelo 2 } \\
\text { Model 2 }\end{array}$ & $\begin{array}{c}\text { Modelo 3 } \\
\text { Model 3 }\end{array}$ & $\begin{array}{c}\text { Regressão } \\
\text { Regression }\end{array}$ & \\
\hline $\begin{array}{l}\text { Fonte de variação } \\
\text { Source of variation }\end{array}$ & $\mathrm{gl}$ & $\begin{array}{c}\text { Quadrado médio } \\
\text { Mean square }\end{array}$ & $\begin{array}{c}\text { Quadrado médio } \\
\text { Mean square }\end{array}$ & $\begin{array}{c}\text { Quadrado médio } \\
\text { Mean square }\end{array}$ & $\beta$ \\
\hline GC & $d f$ & $31,3431^{* *}$ & $48,6563 * *$ & $51,5024 * *$ & \\
IDS & 381 & & & 16,1980 & 0,06743 \\
Linear & 1 & 20,4402 & & 1,1295 & $-0,00001$ \\
Quadrático & 1 & 1,3745 & & 477,6379 \\
PSAJ & 1 & $143,3668 * *$ & $150,7100 * *$ & 0,05220 \\
Linear & 1 & 9,3716 & 12,9575 & 163,5073 \\
Quadrático & & 0,4853 & 0,4673 & 0,4052 & $-0,00002$ \\
$\mathrm{R}^{2}$ & & & & 162,8475 \\
\hline
\end{tabular}

** $(P<0,01)$. *FIV (fator de inflação da variância Modelo 1), GC (grupo contemporâneo), IDS (idade ao sobreano), PSAJ (peso ao sobreano ajustado aos 550 dias), b (coeficiente de regressão).

${ }^{* *}$ FIV (variance inflation factor - Model 1), GC (contemporary group), IDS (yearling age), PSAJ (yearling weight adjusted to 550 days).

R. Bras. Zootec., v.32, n.6, p.1878-1882, 2003 (Supl. 2) 


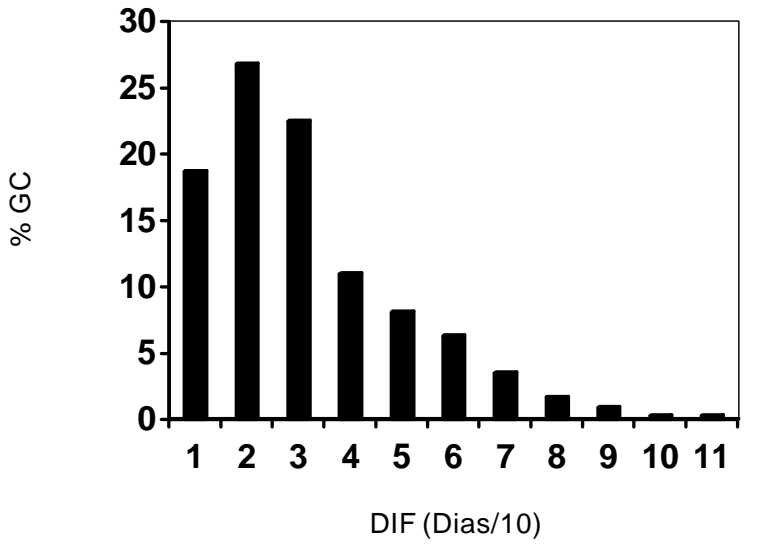

Figura 1 - Freqüência dos grupos contemporâneos ao sobreano (GC), de acordo com as classes de 10 dias de diferença de idade (DIF) entre animais pertencentes ao mesmo GC.

Figure 1 - Frequency of contemporary group (GC), according to 10 days of age difference classes (DIF) among animals belonging to the same GC.

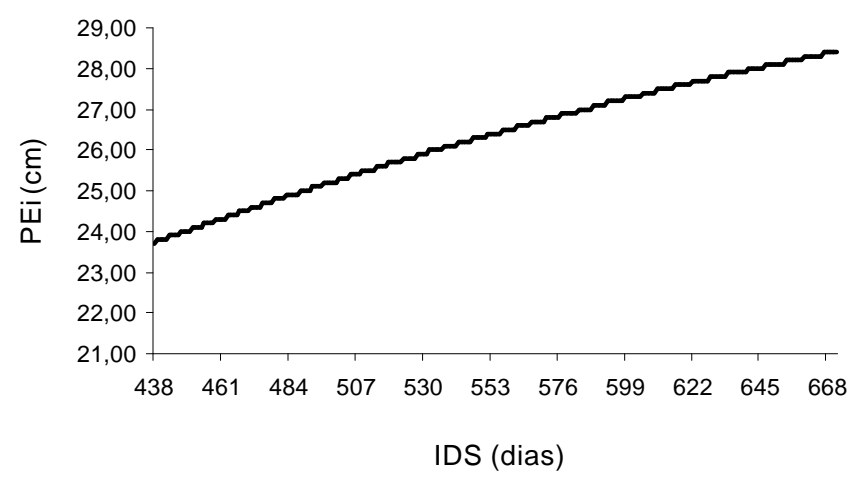

Figura 2 - Perímetro escrotal $\left(P E_{i}\right)$ de bovinos Nelore, predito em função da idade ao sobreano (IDS).

Figure 2 - Predicted scrotal circumference (PE) of Nelore cattle with regarding to yearling age (IDS).

e diminuição da variância residual de $20,94 \%$, o que resultou em uma diferença de 0,07 na estimativa de herdabilidade, representando aumento de $20 \%$. Ao comparar os modelos 1 e 2 (com apenas PSAJ como covariável), tanto a variância genética aditiva quanto a residual diminuíram com a inclusão do peso e idade no modelo. Entretanto, a variância genética diminuiu em apenas $1 \%$, enquanto a variância residual reduziu em $5 \%$, o que resultou em pequeno aumento na herdabilidade estimada para o modelo 1.

Quirino \& Bergmann (1997) observaram que a inclusão do peso como covariável no modelo para análise de perímetro escrotal, em determinadas idades, resultou na diminuição dos componentes de
Tabela 2 - Estimativas dos componentes de variância e da herdabilidade para perímetro escrotal

Table 2 - Estimates of variance components and heritability to scrotal circumference

\begin{tabular}{lcccc}
\hline $\begin{array}{l}\text { Modelos } \\
\text { Models }\end{array}$ & $\sigma_{\mathrm{a}}{ }^{2}$ & $\sigma_{\mathrm{e}}{ }^{2}$ & $\sigma_{\mathrm{p}}{ }^{2}$ & $\mathrm{~h}^{2} \pm \mathrm{ep}$ \\
\hline 1 & 2,2893 & 3,2079 & 5,4972 & $0,42 \pm 0,04$ \\
2 & 2,3113 & 3,3638 & 5,6751 & $0,41 \pm 0,04$ \\
3 & 2,2321 & 4,0579 & 6,2901 & $0,35 \pm 0,03$ \\
\hline
\end{tabular}

Modelo 1- Efeitos linear e quadrático de IDS (idade ao sobreano) e PSAJ (peso ao sobreano ajustado), Modelo 2 - Efeitos linear e quadrático de PSAJ e Modelo 3 - Efeitos linear e quadrático de IDS. $\sigma_{\mathrm{a}}{ }^{2}$ é a variância do efeito genético aditivo direto, $\sigma_{e}{ }^{2}$ é a variância residual, $\sigma_{p}^{2}$ é a variância fenotípica e $h^{2}$ é a herdabilidade da característica e ep - erro-padrão.

Model 1- Linear and quadratic effects of yearling age and yearling weight adjusted to 550 days, Model 2 - Linear and quadratic effect of yearling weight adjusted to 550 days, Model 3 - Linear and quadratic effect of yearling age. $\sigma_{a}^{2}=$ additive variance, $\sigma_{e}^{2}=$ residual variance, $\sigma_{p}^{2}=$ phenotypic variance, $h^{2}=$ heritability coefficient and ep = standard error.

variância genético, de ambiente e fenotípico. Entretanto, dependendo da idade em que o animal foi avaliado, a estimativa de herdabilidade foi inferior à obtida pelo modelo em que o perímetro escrotal não foi ajustado para o peso, para animais da raça Nelore.

Os resultados obtidos no presente trabalho estão de acordo com os descritos por Ortiz-Peña (1998), que verificou aumento da variância genética aditiva e diminuição da variância residual no modelo com o perímetro escrotal ajustado para idade e peso em relação ao modelo que incluiu o perímetro escrotal ajustado apenas para idade.

Vários trabalhos na literatura verificaram que o perímetro escrotal é genética e positivamente correlacionado com o peso corporal em várias idades (Meyer et al., 1991; Alencar et al., 1993; Bergmann et al., 1996). Estes trabalhos mostram que a seleção para aumento do PE deve levar, por resposta correlacionada, ao aumento de peso corporal em várias idades, o que nem sempre é desejável. Nestes casos, talvez seja possível diminuir esta resposta correlacionada selecionando-se com base no PE ajustado para idade e peso corporal.

\section{Conclusões}

O peso e a idade ao sobreano foram importantes fontes de variação para o perímetro escrotal.

De acordo com os parâmetros genéticos estimados, pode-se concluir que o perímetro escrotal é uma característica que deve responder à seleção individual. 


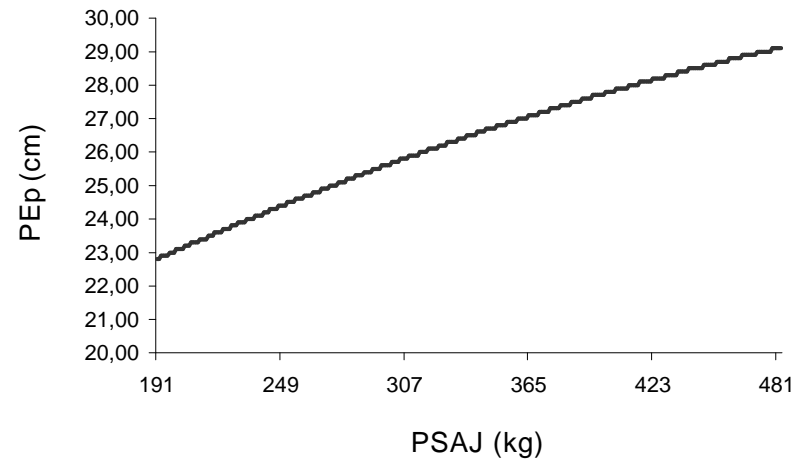

Figura 3 - Perímetro escrotal $\left(\mathrm{PE}_{\mathrm{p}}\right)$ de bovinos Nelore, predito em função do peso ao sobreano ajustado para 550 dias de idade (PSAJ).

Figure 3 - Predicted scrotal circumference (PE) of Nelore cattle with regarding to yearling weight adjusted to 550 days (PSAJ).

O perímetro escrotal ajustado para idade e peso ao sobreano apresentou maior herdabilidade do que quando foi ajustado apenas para idade ou peso e pode ser utilizado como critério de seleção quando se busca, principalmente, maior precocidade sexual.

\section{Literatura Citada}

ALENCAR, M.M.; BARBOSA, P.F.; BARBOSA, R.T. et al. Parâmetros genéticos para peso e perímetro escrotal em touros da raça Canchim. Revista da Sociedade Brasileira de Zootecnia, v.22, n.4, p.572-583, 1993.

BERGMANN, J.A.G.; ZAMBORLINI, L.C.; PROCÓPIO, C.S.O. et al. Estimativas de parâmetros genéticos do perímetro escrotal e do peso corporal em animais da raça Nelore. Arquivo Brasileiro de Medicina Veterinária e Zootecnia, v.48, n.1, p.69-78, 1996.

BOLDMAN, K.G.; Van VLECK, L.D. Derivative-free restricted maximum likelihood estimation in animal models with a sparse matrix solver. Journal of Dairy Science, v.74, p.4337-4343, 1991.

BOLDMAN, K.G.; KRIESE, L.A.; Van VLECK, L. D. et al. A manual for use of MTDFREML. Clay Center: USDA-ARS, 1993. 120p.

BRINKS, J.S.; FIELDS, M.J.; SANDS, R.S. Relationship of scrotal circumference to puberty and subsequent reproductive performance in male and female offspring. Factors affecting calf crop. Boca Raton: CRC Press, 1994. p.363-370.

BRITO, F.V. Influência da idade e peso corporal sobre o perímetro escrotal em touros Hereford - Estimativas de fatores de correção.In: REUNIÃO ANUAL DA SOCIEDADE BRASILEIRA DE ZOOTECNIA, 34., 1997, Juiz de Fora. Anais... Viçosa, MG: Sociedade Brasileira de Zootecnia, 1997. p.130-132.

DAL-FARRA, R.A.; FRIES, L.A.; LOBATO, J.F.P. Fatores de correção do perímetro escrotal para efeitos de idade e peso ao sobreano de tourinhos da raça Nelore. Revista Brasileira de
Zootecnia, v.27, n.6, p.1092-1096, 1998.

FREUND, R.J.; WILSON, W.J. Regression analysis: statistical modeling of a response variable. San Diego: Academic Press, 1998. 444p.

GRASER, H.U.; SMITH, S.P.; TIER, B.A. Derivative-free approach for estimating variance components in animal models by restricted maximum likelihood. Journal of Animal Science, v.64, p.1362-70, 1987.

LATIMER, F.G.; WILSON, L.L.; CAIN, M. F. et al. Scrotal measurements in beef bulls: heritability estimates, breed and test station effects. Journal of Animal Science, v.54, n.3, p.475-479, 1982.

MEYER, K.; HAMMOND, K.; MACKINNON, M.J. et al. Estimates of covariances between reproduction and growth in Australian beef cattle. Journal of Animal Science, v.69, p.3533-3543, 1991.

ORTIZ-PEÑA, C.D. Análise de critérios de seleção para precocidade sexual e de crescimento de bovinos da raça Nelore, no Paraguai. Jaboticabal: Universidade Estadual Paulista, 1998. 143p. Dissertação (Mestrado em Zootecnia) - Universidade Estadual Paulista, 1998.

PATTERSON, H.D.; THOMPSON, R. Recovery of inter-block information when block sizes are unequal. Biometrika, v.58, p.545-54, 1971.

PEREIRA, E.; ELER, J.P.; FERRAZ, J.B.S. Análise genética da relação perímetro escrotal (PE 550)/peso aos 550 dias (PES 550) na raça Nelore. In: SIMPÓSIO NACIONAL DE MELHORAMENTO ANIMAL, 2., 1998, Uberaba. Anais... Uberaba: Sociedade Brasileira de Melhoramento Animal, 1998. p.325-326.

QUIRINO, C.R.; BERGMANN, J.A.G. Herdabilidade do perímetro escrotal ajustado e não ajustado para peso corporal usando modelo animal uni e bivariado. In: REUNIÃO ANUAL DA SOCIEDADE BRASILEIRA DE ZOOTECNIA, 34., 1997, Juiz de Fora . Anais... Viçosa, MG: Sociedade Brasileira de Zootecnia, 1997. p.130-132.

STATISTICAL ANALYSIS SYSTEM - SAS. User's Guide, version 6.12, 4.ed, v.2, Cary: 1998. 842p.

SMITH, S.P.; GRASER, H.U. Estimating variance components in a class of mixed models by restrict maximum likelihood. Journal of Dairy Science, v.29, p.1156-1165, 1986.

TEIXEIRA, R.A.; DIAS, L.T.; ALBUQUERQUE, L.G. et al. Efeitos do peso e idade à desmama e ao sobreano sobre medidas de perímetro escrotal em touros Nelore. In: REUNIÃO ANUAL DA SOCIEDADE BRASILEIRA DE ZOOTECNIA, 35., 1998, Botucatu. Anais... Viçosa, MG: Sociedade Brasileira de Zootecnia, 1998. p.443-445. 